Pacific Journal of Mathematics

HE MODULI SPACE OF GENUS FOUR DOUBLE COVERS OF 


\title{
THE MODULI SPACE OF GENUS FOUR DOUBLE COVERS OF ELLIPTIC CURVES IS RATIONAL
}

\author{
Fabio Bardelli and Andrea Del Centina ${ }^{1}$
}

This paper is devoted to a proof of the rationality of the moduli space of those genus four smooth complex projective curves which are double covers of some elliptic curve. The study of the canonical model of a genus four curve as above allows to reduce the initial moduli problem to a simple one in plane projective geometry; this last formulation leads to compute an explicit representation of a certain group on a vector space and its corresponding field of invariants.

Let $C$ be an irreducible, smooth, projective curve defined over the field of complex numbers.

We call $C$ elliptic-hyperelliptic (e.h. for short) if it admits a degree two morphism $\pi: C \rightarrow E$ onto an elliptic curve. We denote by $\mathscr{M}_{g}^{\text {eh }}$ the moduli space of e.h. curves of genus $g$. The aim of this note is to present a proof of the following:

THEOREM. $\mathscr{M}_{4}^{\text {eh }}$ is rational.

We proceed as follows.

In $\S 1$ the canonical model of a generic e.h. curve $C$ (of genus 4) is shown to be complete intersection of a unique cubic cone $R$ and a unique quadric. By looking at the tangent space to the canonical space at the vertex of $R$, in $\S 2$, we associate to $C$ a pair $(Z, \gamma)$, where $Z$ and $\gamma$ are smooth coplanar curves of degree 3 and 2 respectively, and we are able to show that $\mathscr{M}_{4}^{\text {eh }}$ is birational to

$$
\{(Z, \gamma)\} / \operatorname{PGL}(3) \text {. }
$$

After fixing a quadratic form defining $\gamma$ we can prove that $\{(Z, \gamma)\} / \operatorname{PGL}(3)$ is birational to

$$
H^{0}\left(\mathbf{P}^{1}, \mathscr{O}_{\mathbf{P}^{1}}(6)\right) / G_{l_{0}}
$$

where $G_{l_{0}}$ is a $\mathbf{C}^{*}$-extension of $\mathbf{Z}_{2}$.

\footnotetext{
${ }^{1}$ The authors are members of the GNSAGA of the CNR
} 
In $\S 3$ we compute the representation of $G_{l_{0}}$ on $H^{0}\left(\mathbf{P}^{1}, \mathscr{O}_{\mathbf{P}^{1}}(6)\right)$ and we show that its $G_{l_{0}}$-invariant field is purely transcendental over $\mathbf{C}$, completing the proof of the theorem. We wish to thank the referee for pointing out a mistake that we made in the first draft of the paper and for his helpful comments.

Notation. As usual we denote by $\mathscr{O}_{X}$ and $\Omega_{X}$ the structure sheaf and the canonical sheaf of the irreducible, smooth, projective variety $X$. For any invertible sheaf $\mathscr{F}$ on $X$ we denote by $|\mathscr{F}|^{*}$ the projectivized dual of $H^{0}(X, \mathscr{F})$. We denote by $\mathscr{M}_{g}$ the moduli space of smooth projective curves of genus $g$, and by [C] the point of $\mathscr{M}_{g}$ representing the isomorphism class of the smooth curve $C$ of genus $g$.

If $a$ is an element of a certain group we denote by $\langle a\rangle$ the subgroup generated by $a$.

1. Some geometry of elliptic-hyperelliptic curves. Let us start with some recalls on elliptic-hyperelliptic curves of genus $g \geq 4$ (see [3] for details).

Let $\pi: C \rightarrow E$ be the ramified double cover associated to an elliptic involution $i$ on $C$ ( $E$ is the quotient of $C$ by $\langle i\rangle$ ). To such a cover one can associate the branch locus $B$ on $E$, which is an effective divisor of even degree on $E$, and a half $\mathscr{A}$ of the divisor class of $B$, defined uniquely so that $\pi^{*} \mathscr{A}=\mathscr{O}_{C}\left(\pi^{-1}(B)\right)$. The canonical model $\widetilde{C} \subset\left|\Omega_{C}\right|^{*}$ of $C$ lies on the elliptic normal cone

$$
R=\bigcup_{p \in \widetilde{C}} \overline{P i P}
$$

where $\overline{P i P}$ denotes the line joining conjugated points under $i$. From the natural decomposition

$$
H^{0}\left(C, \Omega_{C}\right) \cong H^{0}\left(E, \Omega_{E}\right) \oplus H^{0}(E, \mathscr{A})
$$

it follows that $R$ is the cone with vertex $\left|\Omega_{E}\right|^{*}$ and projecting the elliptic normal curve $\widetilde{E} \subset|\mathscr{A}|^{*} .\left|\Omega_{E}\right|^{*}$ and $|\mathscr{A}|^{*} \subset\left|\Omega_{C}\right|^{*}$ are the fixed subspaces for the projective transformation of $\left|\Omega_{C}\right|^{*}$ inducing $i$ on $\widetilde{C}$.

$\widetilde{C}$ is also the complete intersection of $R$ and of a suitable quadric containing $\widetilde{C}$.

The branch points on $\widetilde{E}$ are exactly the intersection $|\mathscr{A}|^{*} \cap \widetilde{C}$ so that on $\widetilde{E}$ they are cut by any quadric through $\widetilde{C}$ not containing $\widetilde{E}$.

We set

$$
\mathscr{M}_{g}^{\mathrm{eh}}=\left\{[C] \in \mathscr{M}_{g}: C \text { is e.h. }\right\}
$$


By considering the family of all elliptic normal cones in $\mathbf{P}^{g-1}$ and the family of all quadrics in the same $\mathbf{P}^{g-1}$ one can construct the flat family of all e.h. smooth genus $g$ canonical curves $\mathfrak{F} \rightarrow \mathscr{B}$. Therefore there is a natural morphism $c: \mathscr{B} \rightarrow \mathscr{M}_{g}$ and we get $\mathscr{M}_{g}^{\text {eh }}=\operatorname{Im} c$. It follows that $\mathscr{M}_{g}^{\text {eh }}$ is a subvariety of $\mathscr{M}_{g}$ which by [2] is irreducible and of dimension $2 g-2$. We observe, for the benefit of the reader, that $\mathscr{M}_{g}^{\text {eh }}$ coincides, in the notation of [2] with $S(2,1 ; 1, \ldots, 1)$. Obviously $\mathscr{M}_{g}^{\text {eh }}$ can be regarded as the coarse moduli space for (families of) genus $g$ e.h. curves.

Furthermore [2, Thm. 1] implies immediately the following statement:

1.2. The generic e.h. curve of genus $g \geq 3$ carries exactly one elliptic involution.

Here by "generic" we mean "outside a Zariski closed set."

2. A birational model of $\mathscr{M}_{4}^{\text {eh }}$. Let $U$ be the open subset of $\mathscr{M}_{4}^{\text {eh }}$ corresponding to curves admitting a unique elliptic involution.

For $C$ a canonical curve such that $[C] \in U$ we set:

$$
\begin{aligned}
Q & =\{\text { the unique quadric containing } C\}, \\
R & =\{\text { the unique cubic cone containing } C\}, \\
V & =\{\text { the vertex of } R\}, \\
T C_{V}(R) & =\{\text { the tangent cone to } R \text { at } V\}, \\
T_{V}(R) & =\{\text { the tangent space to } R \text { at } V\} .
\end{aligned}
$$

So in $\mathbf{P} T_{V}(R)$ we find an elliptic curve $Z=\mathbf{P} T C_{V}(R)$ and a conic $\gamma=\mathbf{P}\left(v \in T_{V}(R): l_{v} \cdot Q\right.$ is not reduced), where $l_{v}$ is the line $\{\lambda v\}$.

It is clear that if $C^{\prime}$ is isomorphic to $C$ the pair $\left(Z^{\prime}, \gamma^{\prime}\right)$ gotten from $C^{\prime}$ is projectively equivalent to the pair $(Z, \gamma)$. Hence we get a rational map

$$
U \rightarrow\{(Z, \gamma)\} / \text { PGL(3). }
$$

In order to construct an inverse of the map above we start with a pair $(Z, \gamma)$, we consider the set $\left\{P_{1}, \ldots, P_{6}\right\}=Z \cap \gamma$ and the double cover $C^{\prime}$ of $Z$ branched at $\left\{P_{1}, \ldots, P_{6}\right\}$ with associated line bundle $\mathscr{A}=\mathscr{O}_{Z}(1)$.

It is straightforward to check that this construction gives the inverse we were looking for, so we have proved

LEMMA 2.1. $\mathscr{M}_{4}^{\text {eh }}$ and $\{(Z, \gamma)\} / \operatorname{PGL}(3)$ are birationally isomorphic. 
It is clear that our theorem will follow by proving the rationality of $\{(Z, \gamma)\} /$ PGL $(3)$.

Let $\left(x_{0}: x_{1}: x_{2}\right)$ be homogeneous coordinates in $\mathbf{P} T_{V}(R)=\mathbf{P}^{2}$. We fix once and for all the quadratic form $\Gamma_{0}=x_{0}^{2}-4 x_{1} x_{2}$ in $H^{0}\left(\mathbf{P}^{2}, \mathscr{O}_{\mathbf{P}^{2}}(2)\right)$, let $M$ be its matrix and $\gamma_{0}$ be the conic of equation $\Gamma_{0}=0$.

Since PGL(3) acts transitively on an open dense subset of $\mathbf{P} H^{0}\left(\mathbf{P}^{2}, \mathscr{O}_{\mathbf{P}^{2}}(2)\right)$, we get the following birational isomorphism:

$$
\begin{aligned}
\left\{\left(Z, \gamma_{0}\right)\right\} / \text { Aut } \gamma_{0} & =\mathbf{P} H^{0}\left(\mathbf{P}^{2}, \mathscr{O}_{\mathbf{P}^{2}}(3)\right) / \text { Aut } \gamma_{0} \\
& \cong\{(Z, \gamma)\} / \mathbf{P G L}(3) .
\end{aligned}
$$

Let $G$ be the special orthogonal group of the quadratic form $\Gamma_{0}$ i.e.:

$$
G=\left\{A, 3 \times 3 \text { matrices: } A M^{t} A=M, \operatorname{det} A=1\right\} .
$$

It is a well known fact that:

$$
G \cong \text { Aut } \gamma_{0}
$$

so we get

$$
\left\{\left(Z, \gamma_{0}\right)\right\} / \text { Aut } \gamma_{0} \cong\left\{\left(Z, \gamma_{0}\right)\right\} / G \text {. }
$$

Since $G$ acts on the conic $\gamma_{0} \cong \mathbf{P}^{1}$ and equivariantly on $H^{0}\left(\mathbf{P}^{2}, \mathscr{O}_{\mathbf{P}^{2}}(3)\right)$, we get an induced action of $G$ on $H^{0}\left(\gamma_{0}, \mathscr{O}_{\gamma_{0}}(3)\right)$ and so an action of $G$ on $H^{0}\left(\mathbf{P}^{1}, \mathscr{O}_{\mathbf{P}^{1}}(6)\right)$.

Proposition 2.2. Let $G_{l_{0}}$ be the subgroup of $G$ which fixes the line of equation $x_{0}=0$. Then $\left\{\left(Z, \gamma_{0}\right)\right\} / G$ and $H^{0}\left(\mathbf{P}^{1}, \mathscr{O}_{\mathbf{P}^{1}}(6)\right) / G_{l_{0}}$ are birationally isomorphic.

Proof. We will proceed in several steps.

(1) From the exact sequence

$$
0 \rightarrow \mathscr{I}_{\gamma_{0}}(3) \rightarrow \mathscr{O}_{\mathbf{P}^{2}}(3) \rightarrow \mathscr{O}_{\gamma_{0}}(3) \rightarrow 0
$$

where $\mathscr{F}_{\gamma_{0}}$ denotes the ideal sheaf of $\gamma_{0}$ in $\mathbf{P}^{2}$ (so $\mathscr{I}_{\gamma_{0}}=\mathscr{O}_{\mathbf{P}^{2}}(-2)$ ), we get the exact sequence:

$$
0 \rightarrow H^{0}\left(\mathbf{P}^{2}, \mathscr{I}_{\gamma_{0}}(3)\right) \rightarrow H^{0}\left(\mathbf{P}^{2}, \mathscr{O}_{\mathbf{P}^{2}}(3)\right) \rightarrow H^{0}\left(\gamma_{0}, \mathscr{O}_{\gamma_{0}}(3)\right) \rightarrow 0 .
$$

Let $S=H^{0}\left(\mathbf{P}^{2}, \mathscr{I}_{\gamma_{0}}(3)\right)$ : we remark that $S \cong H^{0}\left(\mathbf{P}^{2}, \mathscr{O}_{\mathbf{P}^{2}}(1)\right)$.

One can see immediately that $S$ is an invariant subspace for the action of $G$ on $H^{0}\left(\mathbf{P}^{2}, \mathscr{O}_{\mathbf{P}^{2}}(3)\right)$.

Therefore by [4, Chap. iv] there is a subspace $W \subset H^{0}\left(\mathbf{P}^{2}, \mathscr{O}_{\mathbf{P}^{2}}(3)\right)$ complementary to $S$ and invariant for $G$. 
Clearly

$$
W \cong H^{0}\left(\gamma_{0}, \mathscr{O}_{\gamma_{0}}(3)\right)
$$

and the action of $G$ on both spaces is equivariant with respect to this isomorphism.

(2) We fix once and for all the linear form $x_{0}$ in $H^{0}\left(\mathbf{P}^{2}, \mathscr{O}_{\mathbf{P}^{2}}(1)\right)$ and we let $l_{0}$ be the line of equation $x_{0}=0$.

Set

$$
\begin{aligned}
& G_{l_{0}}=\left\{A \in G:{ }^{t} A x_{0}=\lambda x_{0}, \lambda \in \mathbf{C}^{*}\right\}, \\
& G_{0}=\left\{A \in G:{ }^{t} A x_{0}=x_{0}\right\} .
\end{aligned}
$$

One can readily see that

$$
G_{0}=\left\{\left(\begin{array}{ccc}
1 & 0 & 0 \\
0 & \alpha & 0 \\
0 & 0 & \alpha^{-1}
\end{array}\right), \alpha \in \mathbf{C}^{*}\right\}
$$

After calling

$$
G_{0}^{\prime}=\left\{\left(\begin{array}{ccc}
-1 & 0 & 0 \\
0 & 0 & \alpha \\
0 & \alpha^{-1} & 0
\end{array}\right), \alpha \in \mathbf{C}^{*}\right\}
$$

one finds that $G_{l_{0}}=G_{0} \cup G_{0}^{\prime}$ and also that $G_{0}^{\prime}=G_{0}\langle m\rangle$ where

$$
m=\left(\begin{array}{ccc}
-1 & 0 & 0 \\
0 & 0 & 1 \\
0 & 1 & 0
\end{array}\right)
$$

We consider the subspace

$$
\mathbf{1} \oplus W=\left\{\alpha \Gamma_{0} x_{0}+w: \alpha \in \mathbf{C}, w \in W\right\} \subset S \oplus W .
$$

$\mathbf{1} \oplus W$ is invariant under the induced action of $G_{l_{0}}$ and we have a (natural) rational map

$$
\phi: \mathbf{P}(\mathbf{1} \oplus W) / G_{l_{0}} \rightarrow \mathbf{P}(S \oplus W) / G .
$$

We claim that $\phi$ is birational.

$\phi$ is dominant: in fact for $\Gamma_{0} l+w \in \mathbf{P}(S \oplus W)$ there is an element $\sigma$ in $G$ such that $\sigma(l)=l_{0}$ and so $\sigma\left(\Gamma_{0} l+w\right)=\Gamma_{0} l_{0}+w^{\prime} \in \mathbf{P}(\mathbf{1} \oplus W)$. $\phi$ is also injective on the set of elements for which $\alpha \neq 0$ : we may assume $\alpha=1$, if $\Gamma_{0} x_{0}+w^{\prime}$ and $\Gamma_{0} x_{0}+w^{\prime \prime}$ are equivalent with respect to $G$, there are $\sigma \in G$ and $\alpha \in \mathbf{C}^{*}$ such that $\sigma\left(\Gamma_{0} x_{0}+w^{\prime}\right)=$ $\alpha\left(\Gamma_{0} x_{0}+w^{\prime \prime}\right)$ in $S \oplus W$.

Since $\sigma\left(x_{0}\right)=\alpha x_{0}, \sigma \in G_{l_{0}}$ and we are done. 
(3) There is an obvious birational map

$$
W / G_{l_{0}} \stackrel{\sim}{\rightarrow} \mathbf{P}(\mathbf{1} \oplus W) / G_{l_{0}}
$$

and by using the isomorphisms

$$
W=H^{0}\left(\gamma_{0}, \mathscr{O}_{\gamma_{0}}(3)\right) \cong H^{0}\left(\mathbf{P}^{1}, \mathscr{O}_{\mathbf{P}^{1}}(6)\right)
$$

(which are equivariant for the $G_{l_{0}}$-action) we conclude the proof.

3. The rationality of $\mathscr{M}_{4}^{\text {eh }}$. To finish the proof of our theorem we have just to prove that $H^{0}\left(\mathbf{P}^{1}, \mathscr{O}_{\mathbf{P}^{1}}(6)\right) / G_{l_{0}}$ is rational.

Let $\left(t_{1}: t_{2}\right)$ be homogeneous coordinates in $\mathbf{P}^{1}$ and consider the Veronese map

$$
\mathbf{P}^{1} \rightarrow \gamma_{0} \subset \mathbf{P}^{2}
$$

given by:

$$
\begin{aligned}
& x_{0}=2 t_{1} t_{2}, \\
& x_{1}=t_{1}^{2}, \\
& x_{2}=t_{2}^{2} .
\end{aligned}
$$

Since the action of $G_{0}$ on $\mathbf{P}^{2}$ is given by:

$$
\begin{aligned}
& x_{0} \mapsto x_{0}, \\
& x_{1} \mapsto \alpha x_{1}, \\
& x_{2} \mapsto \alpha^{-1} x_{2}
\end{aligned}
$$

(for $\alpha \in \mathbf{C}^{*}$ ), we get on the $t_{i}$ 's:

$$
t_{1}^{6-i} t_{2}^{i} \mapsto \alpha^{3-i} t_{1}^{6-i} t_{2}^{i}, \quad 0 \leq i \leq 6,
$$

so that the induced action of $G_{0}$ on $H^{0}\left(\mathbf{P}^{1}, \mathscr{O}_{\mathbf{P}^{1}}(6)\right)$ can be represented by the diagonal matrix:

$$
\left(\begin{array}{ccccccc}
\alpha^{3} & & & & & & \\
& \alpha^{2} & & & & & \\
& & \alpha & & & & \\
& & 1 & & & \\
& & & \alpha^{-1} & & \\
& & & & & \alpha^{-2} & \\
& & & & & & \alpha^{-3}
\end{array}\right)
$$


By the same argument, the action of the element $m \in G_{l_{0}}$ can be represented by the matrix

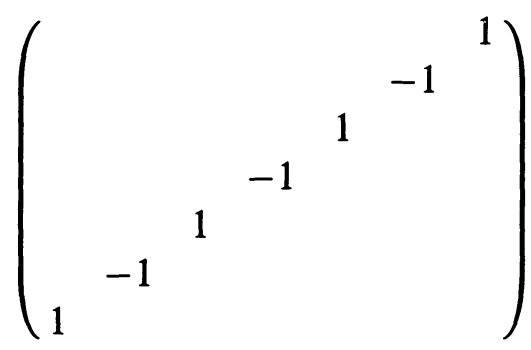

Now let $z_{3-i}=t_{1}^{6-i} t_{2}^{i}$ for $i=0,1,2,3$ and $z_{10-i}=t_{1}^{6-i} t_{2}^{i}$ for $i=4,5,6$ so that the $G_{l_{0}}$-action is given by the standard product of the two matrices above by the vector ${ }^{t}\left(z_{3}, z_{2}, z_{1}, z_{0}, z_{6}, z_{5}, z_{4}\right)$. Then the $G_{0}$-invariant field of $H^{0}\left(\mathbf{P}^{1}, \mathscr{O}_{\mathbf{P}^{1}}(6)\right)$ is

$$
\mathbf{C}\left(w_{1}, w_{2}, w_{3}, w_{4}, w_{5}, w_{6}\right)
$$

where

$$
\begin{aligned}
& w_{1}=z_{0}, \\
& w_{2}=z_{4} z_{3}, \\
& w_{3}=z_{5} z_{2}, \\
& w_{4}=z_{6} z_{1}, \\
& w_{5}=z_{2} z_{1}^{-2}, \\
& w_{6}=z_{3} z_{1}^{-3} .
\end{aligned}
$$

In fact, it is enough to observe that the orbit of an element $w^{\prime}$ with $z_{1} \neq 0$ contains a unique element $w$ with $z_{1}=1$ (which is gotten by acting on $w^{\prime}$ via the element of $G_{0}$ having $\alpha=z_{1}^{-1}$ ) and that the coordinates of $w$ are

$$
\left(z_{3} z_{1}^{-3}, z_{2} z_{1}^{-2}, 1, z_{0}, z_{6} z_{1}, z_{5} z_{1}^{2}, z_{4} z_{1}^{3}\right) \text {. }
$$

The $G_{0}$-invariant functions $w_{1}, \ldots, w_{6}$ given above are gotten easily from these coordinates of $w$.

Now we consider the action of $\langle m\rangle$ on the $w_{i}$ 's. It is immediately seen that $m$ acts as the identity on $w_{2}, w_{3}, w_{4}$, whereas

$$
\begin{aligned}
& m\left(w_{1}\right)=-w_{1}, \\
& m\left(w_{6}\right)=\frac{w_{2}}{w_{4}^{3}} \cdot \frac{1}{w_{6}}, \\
& m\left(w_{5}\right)=-\frac{w_{3}}{w_{4}^{2}} \cdot \frac{1}{w_{5}} .
\end{aligned}
$$


Let us consider the functions

$$
\begin{aligned}
& f_{1}=w_{6}+\frac{w_{2}}{w_{4}^{3}} \cdot \frac{1}{w_{6}}, \\
& f_{2}=w_{5}-\frac{w_{3}}{w_{4}^{2}} \cdot \frac{1}{w_{5}}, \\
& f_{3}=\left(w_{6}-\frac{w_{2}}{w_{4}^{3}} \cdot \frac{1}{w_{6}}\right) w_{1}, \\
& f_{4}=\left(w_{5}+\frac{w_{3}}{w_{4}^{2}} \cdot \frac{1}{w_{5}}\right) w_{1},
\end{aligned}
$$

and

$$
w_{1}^{2}
$$

They are clearly invariant for the action of $\langle m\rangle$; furthermore the following relations hold:

$$
\begin{gathered}
f_{1}^{2}-4 \frac{w_{2}}{w_{4}^{3}}=\left(\frac{f_{3}}{w_{1}}\right)^{2} ; \quad f_{2}^{2}+4 \frac{w_{3}}{w_{4}^{2}}=\left(\frac{f_{4}}{w_{1}}\right)^{2}, \\
\left\{\begin{array}{l}
w_{6}^{2}-f_{1} w_{6}+\frac{w_{2}}{w_{4}^{3}}=0, \\
w_{1}=f_{3} /\left(w_{6}-\frac{w_{2}}{w_{4}^{3}} \cdot \frac{1}{w_{6}}\right), \\
w_{5}=\frac{1}{2}\left(f_{2}+\frac{f_{4}}{w_{1}}\right) .
\end{array}\right.
\end{gathered}
$$

By (3.2) $\mathbf{C}\left(w_{1}, \ldots, w_{6}\right)^{\langle m\rangle} \cong \mathbf{C}\left(w_{1}^{2}, w_{2}, w_{3}, w_{4}, f_{1}, f_{2}, f_{3}, f_{4}\right)$, and by (3.1) this last field is isomorphic to $\mathbf{C}\left(w_{1}^{2}, w_{4}, f_{1}, f_{2}, f_{3}, f_{4}\right)$ which is purely transcendental over $\mathbf{C}$ : thus yielding the proof of our theorem.

\section{REFERENCES}

[1] R. D. M. Accola, Riemann surfaces, theta functions, and abelian automorphism groups, Lecture Notes in Mathematics 483, Springer Verlag 1975.

[2] M. Cornalba, On the locus of curves with automorphisms, Ann. Mat. Pura e Appl. ta (IV), CIL (1987), 135-151. 
[3] A. Del Centina, Remarks on curves admitting an involution of genus $\geq 1$ and some applications, Boll. Un. Mat. Ital. (6) 4-B (1985), 671-683.

[4] J. Fogarty, Invariant Theory, Benjamin 1969.

Received August 12, 1987. Research of the first author was partially supported by MPI $60 \%$ funds.

UNIVERSITÀ

PaVia, ITALY

AND

"U. DinI" Università

FIRENZE, ITALY 



\section{PACIFIC JOURNAL OF MATHEMATICS EDITORS}

\author{
V. S. VARADARAJAN \\ (Managing Editor) \\ University of California \\ Los Angeles, CA 90024-1555-05 \\ Herbert Clemens \\ University of Utah \\ Salt Lake City, UT 84112 \\ THOMAS ENRIGHT \\ University of California, San Diego \\ La Jolla, CA 92093
}

R. FINN

Stanford University

Stanford, CA 94305

Hermann Flaschka

University of Arizona

Tucson, AZ 85721

VAUGHAN F. R. Jones

University of California

Berkeley, CA 94720

Steven Kerckhoff

Stanford University

Stanford, CA 94305

\author{
C. C. MOORE \\ University of California \\ Berkeley, CA 94720
}

MARTIN SCHARLEMANN

University of California

Santa Barbara, CA 93106

HAROLd STARK

University of California, San Diego

La Jolla, CA 92093

\section{ASSOCIATE EDITORS}
R. ARENS
E. F. BECKENBACH
B. H. NeUmanN
F. WoLF
(1904-1989)
K. YosHIDA
(1906-1982)

\section{SUPPORTING INSTITUTIONS}

UNIVERSITY OF ARIZONA
UNIVERSITY OF BRITISH COLUMBIA
CALIFORNIA INSTITUTE OF TECHNOLOGY
UNIVERSITY OF CALIFORNIA
MONTANA STATE UNIVERSITY
UNIVERSITY OF NEVADA, RENO
NEW MEXICO STATE UNIVERSITY
OREGON STATE UNIVERSITY

UNIVERSITY OF ARIZONA

CAIVERSITY OF BRITISH COLUN UNIVERSITY OF CALIFORNIA

MONTANA STATE UNIVERSITY OREGON STATE UNIVERSITY

\author{
UNIVERSITY OF OREGON \\ UNIVERSITY OF SOUTHERN CALIFORNIA \\ STANFORD UNIVERSITY \\ UNIVERSITY OF HAWAII \\ UNIVERSITY OF TOKYO \\ UNIVERSITY OF UTAH \\ WASHINGTON STATE UNIVERSITY \\ UNIVERSITY OF WASHINGTON
}

The Supporting Institutions listed above contribute to the cost of publication of this Journal, but they are not owners or publishers and have no responsibility for its content or policies.

Mathematical papers intended for publication in the Pacific Journal of Mathematics should be in typed form or offset-reproduced (not dittoed), double spaced with large margins. Please do not use built up fractions in the text of the manuscript. However, you may use them in the displayed equations. Underline Greek letters in red, German in green, and script in blue. The first paragraph must be capable of being used separately as a synopsis of the entire paper. In particular it should contain no bibliographic references. Please propose a heading for the odd numbered pages of less than 35 characters. Manuscripts, in triplicate, may be sent to any one of the editors. Please classify according to the 1980 Mathematics Subject Classification (1985 Revision) scheme which can be found in the December index volumes of Mathematical Reviews. Supply name and address of author to whom proofs should be sent. All other communications should be addressed to the managing editor, or Elaine Barth, University of California, Los Angeles, California 90024-1555-05.

There are page-charges associated with articles appearing in the Pacific Journal of Mathematics. These charges are expected to be paid by the author's University, Government Agency or Company. If the author or authors do not have access to such Institutional support these charges are waived. Single authors will receive 50 free reprints; joint authors will receive a total of 100 free reprints. Additional copies may be obtained at cost in multiples of 50 .

The Pacific Journal of Mathematics (ISSN 0030-8730) is published monthly. Regular subscription rate: $\$ 190.00$ a year (12 issues). Special rate: $\$ 95.00$ a year to individual members of supporting institutions.

Subscriptions, orders for numbers issued in the last three calendar years, and changes of address should be sent to Pacific Journal of Mathematics, P.O. Box 969, Carmel Valley, CA 93924, U.S.A. Old back numbers obtainable from Kraus Periodicals Co., Route 100, Millwood, NY 10546.

The Pacific Journal of Mathematics at P.O. Box 969, Carmel Valley, CA 93924 (ISSN 0030-8730) is published monthly. Second-class postage paid at Carmel Valley, California 93924, and additional mailing offices. Postmaster: send address changes to Pacific Journal of Mathematics, P.O. Box 969, Carmel Valley, CA 93924.

\section{PUBLISHED BY PACIFIC JOURNAL OF MATHEMATICS, A NON-PROFIT CORPORATION}




\section{Pacific Journal of Mathematics}

Vol. 144, No. $2 \quad$ June, 1990

George E. Andrews and David M. Jackson, An algebraically derived $q$-analogue of a character sum associated with a class of semiregular

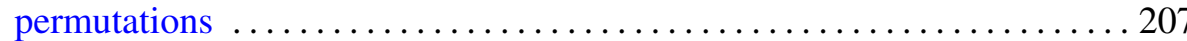

Fabio Bardelli and Andrea Del Centina, The moduli space of genus four double covers of elliptic curves is rational $\ldots \ldots \ldots \ldots \ldots \ldots \ldots \ldots 219$

Young Do Chai, An estimate of the volume of a compact set in terms of its

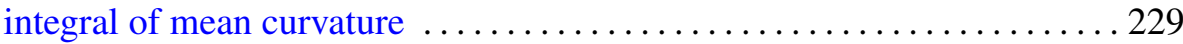

Salvador Comalada, Elliptic curves with trivial conductor over quadratic fields

Kahar El-Hussein, Résolubilité semi-globale des opérateurs différentiels invariants sur les groupes de déplacements $\ldots \ldots \ldots \ldots \ldots \ldots \ldots \ldots . \ldots 259$

David M. Goldschmidt, Classical link invariants and the Burau representation

Liliana Janicka, Radon-Nikodým problem for the variation of a vector measure

Wacław Marzantowicz, An almost classification of compact Lie groups with Borsuk-Ulam properties ............................ 299

Akira Ohbuchi, On the projective normality of some varieties of degree 5 . . 313 Ken'ichi Ohshika, Minimal measured laminations in geometric

3-manifolds

Hal Leslie Smith, A discrete Lyapunov function for a class of linear differential equations

John Samuel Spielberg, Diagonal states on $O_{2} \ldots \ldots \ldots \ldots \ldots \ldots \ldots \ldots$

Thomas Vogel, A note on the sessile drop

Gerold Wagner, On means of distances on the surface of a sphere (lower bounds) 